\title{
Uso de Gamaglobulina Endovenosa en el Púrpura Trombocitopénico Idiopático en el Niño
}

\author{
Dr. Benito González Im. ' : Dra. Patricia Fodor S. ${ }^{1}$; \\ Dra. Walrraud Schuh $O$. \\ Intravenous Gammaglobulin for Idiopathic \\ Thrombocitopenic Purpura.
}

\begin{abstract}
A patient with Cronic thrombocytopenic purpura (ITP) not responding to splenectomy, prednisone and azathioprine in usual doses was treated with intravenous comercial polivalent gammaglobulin $0,4 \mathrm{~g} / \mathrm{kg}$ of body weight per day for five days. Platelet counts reached near nomal values after six days of treatment, remaining within the normal range during 15 days to fall again to abnomal counts in 30 days. However, this time, a new treatment period with immnunosupressors succesfully rose platelet counts to normal, the response persisting indefinitely. No untoward effects of Ig therapy were observed.
\end{abstract}

El Púrpura Trombocitopénico Idiopático del niño (PTI), es una entidad clínica relativamente frecuente cuya causa aún desconoce ${ }^{1}$. Un pequeño porcentaje de estos pacientes especialmente las formas crónicas, se vuelven refractarios a los tratamientos clásicos habituales tales como prednisona, drogas inmunosupresoras y esplenec. tomia ${ }^{2-3}$.

Observaciones recientes, revelan un aumento importante del número de plaquetas en pacientes tratados con gamaglobulina polivalente endove-

1 Servicio de Pediatría, Hospital Luis Calvo Mackenna. nosa en altas dosis, especjalmente pacientes previamente esplectomizados ${ }^{4}$.

Fehr y cols. ${ }^{5}$, demostraron que este tratamiento es también aplicable en algunos pacientes aduitos.

El propósito de esta comunicación, es dar a conocer los resultados obtenidos en un niño con PTI tratado con gamaglobulina endovenosa..

\section{Caso Clínico}

M.V.G., sexo femenino, nacida de embarazo de 34 sermanas de gestación, peso de nacimiento de 3,200 g., talla $51 \mathrm{~cm}$. Episodios de sangra- 
miento $y$ petequias que comenzaron a los 2 años y 7 meses con recuentos de 1.200 plaquetas por $\mathrm{mm}^{3}$, sin causa aparente. En el mielograma dcmostró abundantes megacariocitos. Fue tratada con prednisona en dosis de $2 \mathrm{mg} \times \mathrm{kg} x \mathrm{dia}$ durante ocho meses sin respuesta alguna. Se agregó Azatioprina, durante dos meses.

A pesar del tratamiento continuó con plaquetas bajas, por lo que fue necesario recurrir a esplenectomia a los 3 años y 5 meses de edad.

En el perjodo comprendido entre las edades de 4 y 7 años se le dio prednisona $y$ drogas inmunosupresoras de modo intermitente, persistiendo sus plaquetas bajas con frecuentes sangra. mientos.

A los 7 años fue hospitalizada por malas cundiciones generales y nutritivas, abundantes petequias, equimosis, trombocitopenia de 9.000 plaquetas $\mathrm{x} \mathrm{mm}^{3}$, concentraciones séricas de IgG. IgM, $\lg A$, anticuerpos antinucleares dentro dc rangos normales $y$ se le administ ió gammaglobulina. polivalente durante 5 dias sucesivos $0,4 \mathrm{~g} x$ $\mathrm{kg}$. $x$ día. Cada dosis cortenía $50 \mathrm{mg} \times \mathrm{ml}$ de gammaglobulina solución y fue perfundida a una velocidad de $1-2 \mathrm{ml}$ por minuto.
Se efectuaron controles hematológicos e inmunológicos previos y posteriores al inicio del tratamien to. Ellos demuestran un aumento significativo de los recuentos plaquetarios al sexto día de iniciado el tratamiento. En la Figura 1, se observa el aumento del número de plaquetas que desde una cifra basal de 18.000 por mm $^{3}$ aumentaron a 98.000 por $\mathrm{mm}^{3}$ desde el sexto hasta el décimo quinto dia, para retomar a las cifras basales al cabo de 30 dias.

La concentración inicial de IgG fue de 1.120 $\mathrm{mg} x$ di aumentando a $2.400 \mathrm{mg} \mathrm{x} \mathrm{dl} \mathrm{al} \mathrm{tercer} \mathrm{día}$ de tratamiento, pero después de 15 dias volvió a la basal.

No se observaron efectos secundarios adversos durante el tratamiento. Mientras la paciente mantuvo cifras de plaquetas normales hubo mejoria clínica evidente, con remisión de los signos hemorragicos. A continuación del tratamiento con inmunoglobulina, se repitió un nuevo ciclo can drogas inmunosupresoras, observándose buena respuesta, alcanzando recuentos de 100.000 plaquetas por $\mathrm{mm}^{3}$, que se han mantenido indefinidamente.

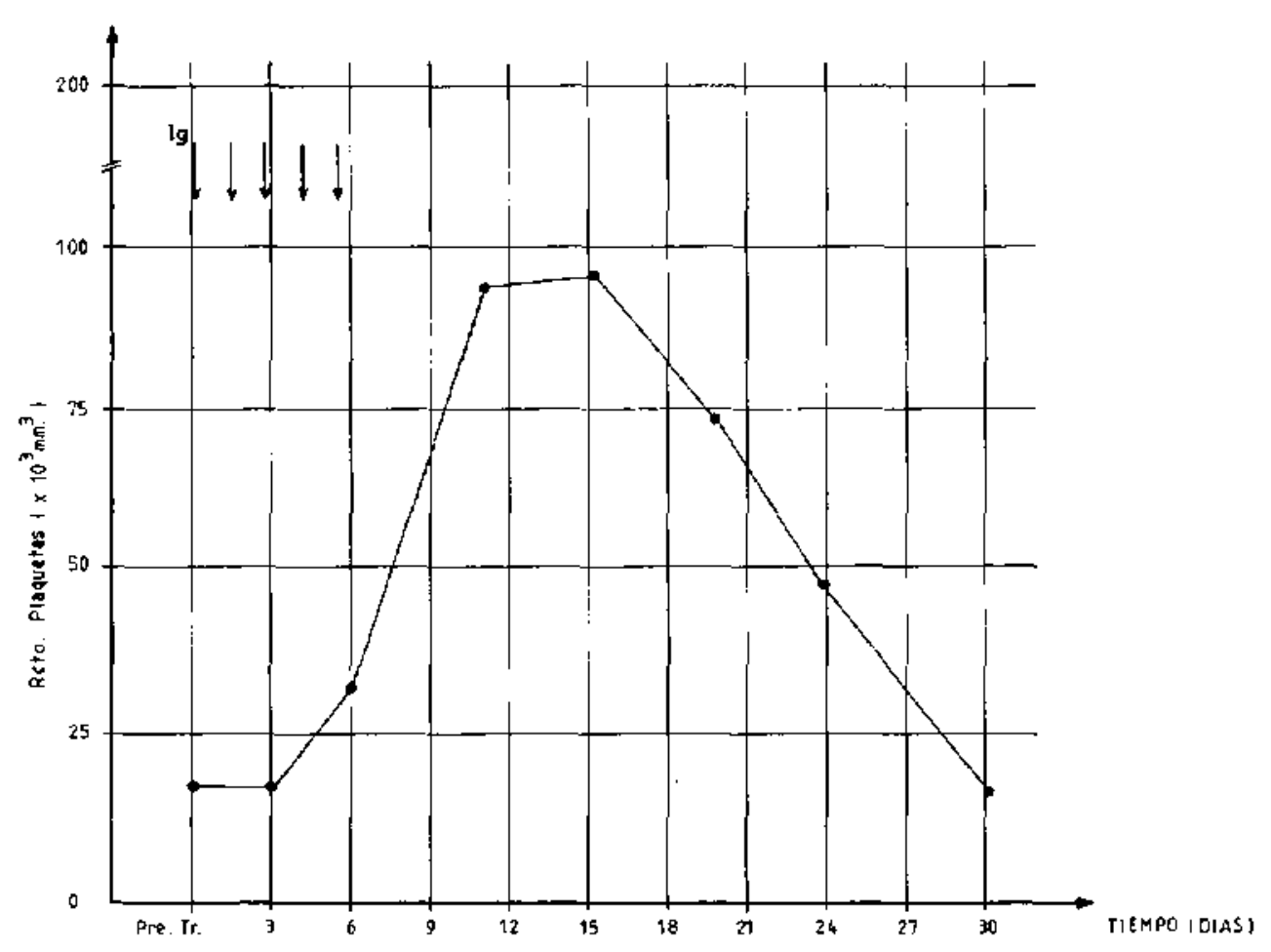

Figura 1: Respuesta a Gammaglobulina Endovenosa en un paciente con PTI. 


\section{COMENTARIO}

El PTI del niño constituye una entidad clínica de causa desconocida. La información existente sugiere un mecanismo autoinmune, dado que se han descrito anticuerpos IgG y complemento en la superficie de las plaquetas en diferentes etapas de la enfermedad, en el niño ${ }^{6-7} y$ en el adulto ${ }^{6-8}$. También se han demostrado complejos innunes circulantes ${ }^{9}$.

La característica principal de la enfermedad es la destrucción de plaquetas por el sistema retículoendotelial especialmente en hígado y bazo; esto explica la mayoría de sus manifestaciones ${ }^{10-11}$.

Los macrófagos, mediante sus receptores $\mathrm{Fc}$, fagocitan plaquetas previamente recubiertas de inmunoglobulinas y complemento, provocando la trombocitopenia. Fehr y cols. ${ }^{5}$, estudiaron la actividad del macrófago aislado en el PTI encontrándola normal. Sugirieron entonces, que la hiperactivjdad del sistema retículoendotelial se debería a alteraciones de origen inmunitario de la superficie plaquetaria, que causarían un secuestro por el macrófago. El mecanismo de acción mediante el cual la gamaglobulina aumenta el núme. ro de plaquetas se desconoce, Se ha planteado como hipótesis el bloqueo de los receptores $\mathrm{Fc}_{\mathrm{c}}$ del sistema retículoendotelial.

Nuestros resultados sugieren que con el uso de gamaglobulina polivalente endovenosa en dosis altas, se puede lograr transitorjamente un aumento significativo del número de plaquetas después de una semana de tratamiento en pacientes con PTI, información que concuerda con comunicaciones previas ${ }^{4-5}$.

Estos estudios preliminares, permiten sugerir el uso de gamaglobulina endovenosa en altas dosis como medida terapéutica en pacientes que requieran rápida normalización del número de plaquetas en situaciones como hemorragia aguda, antes de la esplenectomia u otras intervenciones quirúrgicas. La ausencia de efectos colaterales adversos con dosis elevadas de gamaglobulina la convierten en una buena alternativa terapếutica en casos calificados de PTI.

\section{RESUMEN}

En una paciente conPTI rebelde a tratanientos habituales se administró inmunoglobulina endovenosa en altas dosis. Nuestros resultados sugieren que con este tratamiento se puede lograr un aumento significativo del número de plaquetas al cabo de la primera semana de tratamiento; manteniéndose este efecto por 15 días. No se observaron efectos colaterales adversos.

\section{REFERENCIAS}

${ }^{1}$ Baldini, $M$. Idiopathic trombocy topenic purpura. N. Eng. J. Med. 274: 1245, 1966.

2 Mc. Williams, N.B.; Maturer, H.M.: Acute Idiopathic thrombocy topenic purpura in children. A., J. Hematol. $7: 87,1979$.

${ }^{3}$ Mc. Millan R.: Chronic jdiopathic thrombocy topenic purpura. N. Engl. J. Med. 304: 1135, 1981.

4 Imbach, P.; Baradun, S., D'Apuzzo, V' et at: High dose intravenous gammaglobulin for idiopathic thrombocy topenic purpura in childiood. Lancet 1 : $1228,1981$.

${ }^{5}$ Fehr, l.; Hofmenn $V_{+}$Xappeler, $V_{\text {: }}$ Transient reversal of thrombocytopenia in idiopathic thrombocy to penic purpura by high-dose intravenous gammaglobulin N. Engl. J. Med, 30 (21): 1254, 1982 ,

6 Luiken, G.A.; Mc. Millan R.; Lightsey, A.L. et al: Platelet associated $\lg G$ in immune thjombocy topenic purpura. Blood 50: $317,1977$.

7 Mc. Wiltams, N.B; Mower, H.M:: Acute idiopathic thrombocytopenic purpura in children. Am. J. Hematol. 7: 87, 1979 .

B Karpatkin, S.: Autoimmune thrombocytopenic purpura. Blood S6: 329, 1980.

9 Trent, R.J.: Clancy. R.L.; Dariis, V.; Basten, A.: Immune Complexes in thrombocytopenic patients: cause or effect. Br. J. Haematol. 44: 645, 1980.

10 Salky, N.K, Mills, D, Di Luzio, N.R.: Activity of the reticuloendothelial system in disease of altered immunity. J. Lab. Clin. Med. 66: 952, 1965.

I1 Frank, M.M.; Schreiber, A.D.; A thinson, J.P. Jafre, C.J.: Pathophysiology of immune hemolytic anemia. Ann. Intern. Med. 87: 210, 1977. 